\title{
Diskussionspapier des BfDI für eine Konzeption der Stiftung Datenschutz
}

Mit der Bereitstellung von Haushaltsmitteln in Höhe von zehn Millionen Euro hat die Bundesregierung die Voraussetzungen geschaffen, um die Stiftung Datenschutz noch in diesem Jahr zu errichten. Nun gilt es, dass im Koalitionsvertrag skizzierte Aufgabenfeld der künftigen Stiftung, nach welchem die Stiftung Produkte und Dienstleistungen auf ihre Datenschutzfreundlichkeit prüfen, Bildung im Bereich des Datenschutzes stärken, den Selbstdatenschutz durch Aufklärung verbessern und ein Datenschutzaudit entwickeln soll, mit Leben zu füllen.

Damit von der Stiftung Datenschutz die von den Regierungsparteien intendierte Stärkung des Datenschutzes ausgehen kann, sind bereits bei ihrer konzeptionellen Ausgestaltung die notwendigen Rahmen- und Strukturbedingungen zu schaffen. Ihr dauerhafter Erfolg wird dabei nicht nur vom Vertrauen in die Unabhängigkeit der Urteilskraft und Arbeit der Stiftung abhängen, sondern auch davon, wie das Verhältnis der Stiftung zu den Datenschutzaufsichtsbehörden ausgestaltet sein wird.

Die der Stiftung zugedachten Aufgaben weisen starke Bezugspunkte zu der Arbeit der Datenschutzbehörden auf. Dies tritt am deutlichsten bei der Bildung und der Verbesserung des Selbstdatenschutzes durch die Öffentlichkeitsarbeit - klassischen Themengebieten der Aufsichtsbehörden - zu Tage, gilt aber auch für die übrigen Tätigkeitsbereiche, weil abzusehen ist, dass sich Unternehmen gegenüber den Aufsichtsbehörden auf positive (Vergleichs-)Tests und Zertifizierungen der Stiftung Datenschutz berufen werden. Stiftung und Datenschutzaufsichtsbehörden müssen daher eng zusammenarbei- ten, wenn divergierende Entscheidungen und ein daraus resultierender Vertrauensverlust verhindert werden sollen. Eine intensive und gut organisierte Zusammenarbeit ist zugleich ein Gebot der Effizienz und der Schonung von Ressourcen.

Auf die Erfordernisse der Unabhängigkeit der Stiftung und der engen Zusammenarbeit mit den Aufsichtsbehörden hat bereits die 80. Konferenz der Datenschutzbeauftragten des Bundes und der Länder im November 2010 hingewiesen. Der Bundesbeauftragte für den Datenschutz und die Informationsfreiheit betont in seinem Diskussionspapier zur Stiftung Datenschutz beide Aspekte.

Das Diskussionspapier bezieht dabei die positiven Erfahrungen mit den seit Jahrzehnten bewährten Strukturen der Stiftung Warentest ein. Dies gilt etwa bei der Frage, unter welchen Voraussetzungen die Annahme von Spenden privater Dritter und Unternehmen zulässig ist, ohne dass hierdurch die finanzielle Unabhängigkeit der Stiftung gefährdet wird. Wie bei der Stiftung Warentest sollte zudem gewährleistet sein, dass die Bundesrepublik Deutschland als Stifterin bestimmenden Einfluss auf die Besetzung des Vorstands und Verwaltungsrats hat und Interessenkonflikte, insbesondere Konflikte mit Unternehmensinteressen, ausgeschlossen sind. Die Beteiligung der Interessengruppen aus Wirtschaft, Verbraucher- und Datenschutz in dem Beratungsgremium der Stiftung findet sich in ähnlicher Weise im Kuratorium der Stiftung Warentest und kann daher als Blaupause für die Stiftung Datenschutz verwendet werden.

In anderen Bereichen muss die Konzeption der Stiftung Datenschutz neue Wege beschreiten. Das gilt zum Beispiel für die
Finanzierung mit Einnahmen aus der Vergabe von Gütesiegeln und Auditierungen. Eine nachhaltige und angemessene Finanzierung, für die die Bundesrepublik Deutschland als Stifterin besondere Verantwortung trägt, muss gewährleisten, dass Personal und Kernbestand der Stiftungstätigkeit aus öffentlichen Mitteln bestritten werden können, damit der Fortbestand der Stiftung nicht von den wenig planbaren Einnahmen aus der Auditierungstätigkeit abhängt. Andernfalls drohen Gefälligkeitsgutachten ohne datenschutzrechtlichen Mehrwert.

Mit der Beteiligung der Aufsichtsbehörden im Stiftungsbeirat wird zudem die Kooperation zwischen der Stiftung Datenschutz und den Datenschutzbehörden institutionell verzahnt. Das Diskussionspapier schlägt eine Kombination aus frühzeitiger Information, einer Plausibilitätskontrolle und einem Widerspruchsrecht des Beirats gegen die Vergabe der Zertifizierung bzw. die Durchführung einer Testreihe vor. Dadurch, dass die Leitungsorgane der Stiftung den Widerspruch des Beirats überstimmen können, bleibt die Entscheidungshoheit und Verantwortung allerdings bei der Stiftung. Die Testergebnisse und Gütesiegel können im Umkehrschluss daher auch die Aufsichtsbehörden nicht binden.

Das Diskussionspapier ist auf der Webseite des BfDI (www.bfdi.bund.de) unter Pressemitteilungen abrufbar.

\section{Jost Onstein}

Referent beim Bundesbeauftragten für den Datenschutz und die Informationsfreiheit (BfDI) 